\title{
Management of refractory complex partial seizures: current state of the art
}

\author{
This article was published in the following Dove Press journal: \\ Neuropsychiatric Disease and Treatment \\ 25 May 2010 \\ Number of times this article has been viewed
}

\section{David M Treiman \\ Division of Neurology, Barrow Neurological Institute, St. Joseph's Hospital, and Medical Center Phoenix and Arizona State University, \\ Tempe, Arizona, USA}

Correspondence: David M Treiman Barrow Neurological Institute, 350 West Thomas Road, Phoenix, AZ 850 I3, USA Tel + I 602406692 I

Fax + I 602406716 I

Email dtreiman@chw.edu

\begin{abstract}
Diagnosis of complex partial epilepsy is based on the clinical history, and laboratory tests, including EEG and neuroimaging studies, corroborate the diagnosis. The goal of epilepsy management is to make the patient completely seizure-free without drug-induced side effects, even in the patient with refractory complex partial seizures. Frequently this can be accomplished by choice of the optimal antiepileptic drug (AED) or a combination of drugs, the use of strategies to maximize the effectiveness of drug treatment, or by surgical removal of the seizure focus. Currently there are five "classical" first-line AEDs and 11 new AEDs available in the US and in many other countries for the treatment of localization-related epilepsy. The current state of the evidence is that no AED is clearly superior to other AEDs in the management of refractory complex partial seizures. Therefore the choice of which drug to use in an individual patient has to be based on other considerations, including the potential adverse reactions that may occur in that patient. There are a number of strategies for optimal use of AEDs in the management of refractory complex partial seizures. These include verification of the diagnosis of epilepsy and classification of specific seizure types, use of monotherapy if possible but polytherapy if necessary, starting with a low dose and raising it slowly but, until complete seizure control is achieved, pushing to the maximum tolerated dose, changing timing of dosing to reduce toxicity, using pharmacokinetic principles to fine-tune AED doses, adjusting dose for drug-drug interactions, and never giving up in the pursuit of better seizure control. Resection of the seizure focus can be curative in the majority of patients with seizures localized to one mesial temporal lobe. Success rates for resection of extratemporal seizure foci are lower. Vagus nerve stimulation (VNS) devices result in a significant reduction of seizure frequency in many patients, but patients rarely become completely seizure-free as a result of VNS device implantation. Management of refractory complex partial seizures continues to improve with the identification of new drugs and the development of new approaches to their control and cure.
\end{abstract}

Keywords: complex partial seizures, localization-related epilepsy, refractory, antiepileptic drugs, management

\section{Introduction}

Epilepsy is the second most common neurologic disorder after headache. At least 50 million people in the world suffer from recurrent nonprovoked seizures, ${ }^{1}$ with age-adjusted prevalence rates of $0.2 \%$ to $4.1 \%$, depending on the population studied. ${ }^{2}$ Fundamentally, there are two types of epilepsy, primary (or idiopathic) generalized epilepsy and localization-related epilepsy. Primary generalized epilepsy is considered to be genetic in etiology, whereas most localization-related epilepsy is presumed to be the result of a cerebral insult, even though the insult cannot be determined in about half of all epileptic patients, regardless of age. ${ }^{3-8}$ However, recent developments in 
imaging technology have reduced this figure in children and young adults with refractory epilepsy, where malformations of cortical development have been implicated in about $25 \%$ of such cases. ${ }^{9-13}$

Localization-related epilepsy is manifested as one or more of three types of seizures: simple partial, complex partial, and partial onset with secondary generalization. The specific seizure type exhibited by a person with localization-related epilepsy is determined by many factors, including choice and dose of antiepileptic drugs, and may vary in the same individual as a result of changes in both the internal and external environment. Localization-related epilepsy accounts for about $60 \%-75 \%$ of all epilepsy, and more than half of such patients experience complex partial seizures. ${ }^{14,15}$ Only about half of patients with complex partial seizures have epilepsy that can be fully controlled with medication; the other half have seizures while on antiepileptic drugs, or experience significant side effects in order to gain complete seizure control. From these statements, it should be apparent that there are more than 7.5 million people in the world with refractory complex partial seizures. This article will discuss recent advances and strategies in the management of refractory complex partial seizures. Seizures are generally considered refractory when two are more antiepileptic drugs have failed to control the seizures. For research purposes, full control has been defined as complete cessation of seizures for at least one year. ${ }^{16,17}$

\section{Epidemiology}

The incidence of epilepsy overall is highest in infancy and early childhood, and in the elderly. ${ }^{3,8}$ However, this U-shaped distribution is not true in localization-related epilepsy, where the incidence is around 20/100,000/year from birth to 60 years of age, after which the incidence rises dramatically. ${ }^{8}$ In a UK study, the incidence of epilepsy (almost all of which was localization-related) increased from 76/100,000 in people 60-69 years of age to 159/100,000 in those 80 years and older. ${ }^{18}$ This dramatic rise in new onset epilepsy in the elderly is largely attributable to cerebrovascular disease and degenerative disorders, such as Alzheimer's disease. ${ }^{19,20}$ These numbers may represent an underestimate of the true incidence, because in a number of epidemiologic studies of epilepsy it is likely that many secondarily generalized tonic-clonic seizures have been labeled as generalized, rather than partial onset. Furthermore, the incidence of epilepsy is clearly higher in developing countries than in industrialized countries. ${ }^{21}$ Nonetheless, it is worthwhile to review further what is known about the epidemiology of localization-related epilepsy in general and, to the extent possible, of intractable complex partial seizures.

Four studies have reviewed the epidemiology of localization-related epilepsy.,14,22-24 Over a 50-year period from 1934 to 1984,880 people were diagnosed with epilepsy in Rochester, Minnesota (a predominantly Caucasian middleclass community). Fifty-seven percent had localization-related epilepsy and, of these, two-thirds had complex partial seizures. The age-adjusted incidence of individuals with complex partial seizures was 16/100,000/year. ${ }^{3}$ In Southwest France the overall incidence of symptomatic localization-related epilepsy was $17.1 / 100,000$ but $33.2 / 100,000$ in those aged 60 or older. ${ }^{23,24}$ In a Danish study of 1508 consecutive adults, $73.5 \%$ had localization-related epilepsy, and individuals with complex partial seizures accounted for $38.2 \%$ of all cases. ${ }^{14}$ Patients with complex partial seizures accounted for $21.4 \%$ of the cases in a recent Icelandic study but nearly half of the cases were listed as generalized, not otherwise specified. ${ }^{8}$

The etiology of localization-related epilepsy is highly dependent on the age of onset. In all age groups, the etiology cannot be determined in more than half $(55 \%-89 \%)$ of all individuals with epilepsy. ${ }^{15}$ When etiology can be determined, congenital anomaly is the largest etiologic category in children. In young adults the etiology is unknown in more than $75 \%$ of patients, but head trauma is the largest single etiologic category. In adults aged 35-64 years, head trauma, vascular insults, and tumors are all important etiologies, whereas cerebrovascular disease and degenerative disorders account for most of the identifiable etiologies in localization-related epilepsy in individuals 65 years of age or older. ${ }^{19}$ Advances in neuroimaging techniques have improved understanding of the etiology of localizationrelated epilepsy. Imaging has allowed the identification of the cause of epilepsy in many patients, especially children previously considered to have cryptogenic epilepsy. For instance, Kuzniecky et $\mathrm{al}^{10}$ reported that $25 \%$ of children undergoing surgery for medically intractable partial seizures had magnetic resonance imaging (MRI) and pathologic findings of cerebral developmental anomalies.

Traumatic brain injury is an important cause of localization-related epilepsy, especially in military personnel. Traumatic brain injury has become known as the signature injury in the Iraq and Afghanistan conflicts because the use of body armor and the frequency of blast injuries have allowed many soldiers to sustain but survive head injuries. As many as $50 \%$ of individuals with severe head injuries, especially penetrating head injuries, may develop localization-related epilepsy, most often within the first two years. ${ }^{25}$ 


\section{Clinical features}

Localization-related epilepsy is manifested as simple partial seizures, complex partial seizures, and partial onset seizures that become secondarily generalized. All start from a cortical seizure focus. Simple partial seizures remain sufficiently localized that there is no impairment of consciousness, and the clinical features of the seizure are a reflection of the location of the seizure focus. Complex partial seizures spread sufficiently to engage portions of the opposite hemisphere, and thus result in at least some impairment of consciousness, ie, decreased contact with the environment. Secondarily generalized seizures start from a cortical focus, but spread to the diencephalon and the opposite hemisphere to produce complete impairment of consciousness and generalized tonicclonic convulsions.

The clinical semiology of complex partial seizures depends on the location of the cortical seizure focus. The temporal lobe is the most common site of origin of complex partial seizures. ${ }^{26,27}$ About $80 \%$ of patients with localization-related epilepsy have temporal lobe seizures. ${ }^{28}$ Complex partial seizures of mesial temporal origin are frequently associated with mesial temporal sclerosis on MRI studies and often begin with an aura. Most common are viscerosensory sensations such as a rising sensation from the abdomen, or a sense of fear. Déjà $v u$ and olfactory sensations are less common. Oro-alimentary automatisms (lip smacking, chewing) are common. Head turning to the ipsilateral side, manual automatisms on the ipsilateral side, or dystonic posturing of the contralateral upper extremity may help lateralize the seizure focus. ${ }^{29}$ Postictal confusion usually lasts at least several minutes, after which there is gradual recovery. Typically, the patient is amnestic for events during the complex partial seizure. However, many patients insist that they are able to perceive their environment during complex partial seizures. This likely is because they remember the simple partial portion of the seizure, which they perceive as the complete seizure. Complex partial seizures of lateral or other neocortical temporal origin probably constitute less than $10 \%$ of temporal onset seizures. ${ }^{27}$ It has been suggested that neocortical temporal lobe seizures most commonly begin with sensory illusions or auditory hallucinations, rapid loss of contact with the environment, early contralateral dystonic posturing without oro-alimentary automatisms, shorter seizure duration, and frequent secondary generalization. ${ }^{27}$ Maillard et $\mathrm{al}^{30}$ have described three electrophysiologic subtypes of temporal lobe epilepsy.

Frontal lobe onset complex partial seizures are most commonly nocturnal. Typically they are abrupt in onset, short in duration, and have little or no postictal confusion. Behavior can be explosive and bizarre. ${ }^{31}$ Because of the bizarre behavior and frequent difficulty recording unequivocal ictal discharges on the EEG (because of large areas of frontal cortex inaccessible to surface electrodes), such seizures may at times be considered psychogenic nonepileptic seizures. However, the stereotyped nature of such seizures in a given patient, regardless of how bizarre the behavior is, should alert the physician to the likelihood of frontal lobe seizures. Characteristics of frontal lobe seizures may vary considerably, depending on location of the seizure focus within the frontal lobe. Characteristics of seven different types of frontal lobe seizures have been detailed in the International Classification of Epilepsies and Epileptic Syndromes. ${ }^{31}$

Occipital lobe origin complex partial seizures are much less common that those of temporal or frontal origin, and are characterized by elementary or complex visual hallucinations or illusions. Forced blinking or eyelid flutter at the beginning of occipital origin seizures has also been described. The clinical features of occipital lobe complex partial epilepsies have recently been reviewed. ${ }^{27}$

Parietal lobe epilepsy is the least common of the localization-related epilepsies. Partial onset seizures of parietal origin consist of sensory alterations, visuospatial disorientation, or apraxias. Seizures arising from or spreading to the parietal eye field may result in ipsilateral movement of the eyes, in contrast with seizures engaging the frontal eye field, which drive the eyes contralateral to the seizure focus. ${ }^{32}$

Diagnosis of complex partial seizures is based on the clinical history, as is true when dealing with all types of epilepsy. Fundamentally epilepsy is a clinical diagnosis. Laboratory tests, including EEG and imaging studies, corroborate the clinical diagnosis. Table 1 presents characteristic features of epileptic seizures, which by history taking enable their differentiation from syncopal spells or psychogenic events. Table 2 presents a series of questions useful in making the diagnosis of an epileptic seizure by history. Deacon et $\mathrm{al}^{33}$ studied the accuracy of clinical histories taken by epileptologists in the identification of seizures in 88 patients with suspected refractory complex partial seizures. They reported

Table I Characteristics of epileptic seizures

- Spontaneous brief episodes of abnormal behavior

- Not time-linked to psychologic precipitants

- Discrete, time-limited events with an identifiable onset and termination

- Predictable evolution of behavior during the seizure

- Progressive recovery of function after the seizure

- Stereotyped from event to event 
Table 2 Historical information helpful for diagnosis of epilepsy

- Detailed second-by-second description of the spell - what happened first, second, etc?

- Loss of consciousness? When in relation to motor activity?

- Bilateral involvement before loss of consciousness (may occur in frontal lobe seizures)?

- Convulsive activity? What and where?

- Tongue biting, incontinence, postictal muscle soreness (may also occur with convulsive syncope or psychogenic events)?

- Stare, automatisms, of what type?

- Nature of onset, cessation?

- Duration of each behavior (ictal events, postictal)?

- Any elements suggesting syncope (pallor, diaphoresis, dimming of vision, rapid recovery)?

- Any elements suggesting psychogenic event (variable behavior, prolonged duration, brief pauses during "convulsive" activity, eyes closed during the event)?

sensitivity for seizure identification of $96 \%$, but specificity of only $50 \%$. Accuracy for complex partial seizures and generalized seizures was higher than for simple partial seizures. Misidentification occurred only with simple partial seizures and nonepileptic events.

The history is also critical in the search for the etiology of the patient's epilepsy. It is essential to review the patient's medical history in sufficient detail to identify possible risk factors for epilepsy. This is especially important if the etiology is one that itself requires treatment and, ideally, can be corrected.

The purpose of the neurologic examination in evaluating the patient with possible epileptic seizures is two-fold. First, the presence of even subtle abnormalities on the neurologic examination adds support to the likelihood that the patient's "spells" are epileptic seizures. Second, identification of lateralizing abnormalities on the neurologic examination allows prediction, to some extent, of the location of the epileptic focus. This is also done by including specific questions regarding seizure semiology when taking the history from patients and observers, and looking for such signs on video recordings of the seizures either from home videos or on an epilepsy monitoring unit (EMU).

Most lateralizing signs are contralateral to the seizure focus and include unilateral clonic activity, tonic activity in frontal origin seizures (but not temporal origin seizures), dystonic posturing, and version (forced deviation of head and eyes). Asymmetric tonic posturing producing a "figureof-four" sign consists of flexion of the ipsilateral arm and rigid extension of the contralateral arm. The last clonic jerk is almost always on the side ipsilateral to the seizure focus, and unilateral postictal paralysis is always contralateral. ${ }^{29}$ Unfortunately, none of these signs is $100 \%$ infallible, so such signs need to be used together to build a confident picture of lateralization, and need to be considered only as suggestive of the likely lateralization of seizure origin. Chung et a ${ }^{34}$ recently described a highly reliable sign with which to differentiate between epileptic seizures and psychogenic nonepileptic events. Almost all (94\%) patients with epileptic seizures keep their eyes open during the seizure, whereas almost all (99\%) patients with nonepileptic events close their eyes during the seizure.

The electroencephalogram (EEG) is an essential element in the evaluation of patients with possible epilepsy, but finding epileptiform discharges on the EEG is not essential for the diagnosis. Even in adults with established epilepsy, only $29 \%$ to $55 \%$ have interictal spikes on their first EEG, although $80 \%$ to $90 \%$ will eventually show interictal spikes on repeated EEG examinations. ${ }^{35}$ In localization-related epilepsy, finding an interictal spike focus, especially in the anterior or midtemporal regions, indicates cortical irritability or hyperexcitability, and strongly suggests the presence of a seizure focus in that location. Focal slowing, indicative of underlying structural damage, may also localize a seizure focus, but is much less specific than a spike or sharp wave focus. The likelihood of observing interictal spikes or sharp waves can be increased significantly by recording 30 minutes of sleep; other EEG activation procedures do not often reveal spikes or sharp waves not present during the routine recording.

Ictal discharges on the EEG are usually observed on an EMU, except on infrequent occasions when a seizure is captured on a routine EEG. Seizures recorded on EMU have the advantage that behavior recorded on video (seizure semiology, as described above) can be correlated with EEG changes. Almost all patients with localization-related epilepsy who are evaluated on an EMU have medically refractory seizures, so studies have not differentiated between ictal EEG findings in patients with medically responsive and medically refractory seizures. Ictal discharges recorded from scalp electrodes can vary in appearance, beginning with low-voltage fast activity or rhythmic slowing, or sometimes flattening of the EEG. There may be a heralding spike at onset, and interictal spikes may increase or decrease in frequency before a partial onset seizure. Onset can be quite focal in onset, begin simultaneously over most of one hemisphere, or begin diffusely over most electrodes. Characteristically, there is an evolution of the EEG changes, often with an increase in amplitude and decrease in frequency as the seizure progresses and, if the 
seizure has begun focally, spread to engage other cortical areas. As a generalization, seizures that impair consciousness exhibit associated EEG changes in scalp recordings, although frontal lobe origin complex partial seizures may not. Seizures that do not impair consciousness (simple partial seizures) may not exhibit EEG changes. Only 21\%-61\% exhibit a scalp EEG correlate. ${ }^{36-38}$ This is because the neuronal hypersynchronous discharges of the simple partial seizure may remain sufficiently localized that EEG changes are attenuated between cortex and scalp. The minimal size of a hypersynchronous cortical discharge that can be detected as a spike by scalp electrodes is $6 \mathrm{~cm}^{2},{ }^{39}$ but a $10 \mathrm{~cm}^{2}$ area of activation is much more likely to be detected on the surface as a spike or ictal rhythm, and synchronous activation of $10-20 \mathrm{~cm}^{2}$ of gyral cortex is common. ${ }^{40,41}$

Neuroimaging has become of increasing importance in the evaluation of refractory complex partial seizures with the development of high resolution MRI, new imaging protocols, and nuclear medicine procedures, such as positron emission tomography scanning, and ictal and interictal single positron emission computerized tomography (SPECT). More recently, subtraction ictal single-photon emission tomography coregistered to magnetic resonance imaging (SISCOM) has enhanced the power of SPECT. These new techniques have led to significant improvement in the ability to identify the ictal onset zone. For instance, it has been suggested that SISCOM can reveal areas of hyperperfusion or hypoperfusion in up to $80 \%$ of patients with intractable seizures and may also predict operative outcome. ${ }^{42,43}$

MRI continues to be the most sensitive and specific imaging technique for localization-related epilepsy. Detection of abnormalities (atrophy, increase in signal) can be enhanced by use of specific "seizure" protocols, which orient the image in the oblique coronal plane to best visualize the amygdalar and hippocampal structures. The introduction of increasingly powerful magnets has improved resolution as well. Hippocampal atrophy can best be seen using $\mathrm{T}_{1}$ - weighted imaging, whereas increased signal brightness can best be appreciated with $\mathrm{T}_{2}$-weighted imaging or fluid-attenuated inversion recovery (FLAIR) sequences. Quantitative volumetric studies can be helpful for the detection of hippocampal atrophy, but are labor intensive and only available in a few centers.

\section{Medical treatment}

The goal of epilepsy management is to make the patient completely seizure-free. This is the goal of management of refractory complex partial seizures as well, and not infrequently can be achieved by the choice of the optimal AED or a combination of drugs, the use of strategies to maximize the effectiveness of drug treatment, or by surgical removal of the seizure focus.

Currently there are five "classical" first-line AEDs and 11 new AEDs available in the US and in many other countries (Table 3). Unfortunately, few direct comparisons provide data with which to determine the relative effectiveness of these drugs. Two US Veterans Administration Cooperative studies compared the classical AEDs in the treatment of new onset partial onset seizures in adults, using a randomized doubleblind design. The principal outcome measure was retention on the drug (ie, the drug was sufficiently effective and sufficiently free of side effects that the patient and investigator agreed to continue that drug). In the first study, phenytoin (PHT) and carbamazepine (CBZ) were superior to phenobarbital (PB) and primidone (PRIM) in patients with mostly simple and/or complex partial seizures. ${ }^{44}$ In the second study there was no difference in retention, but CBZ was superior to divalproex sodium in the treatment of complex partial seizures in four of five secondary measures. ${ }^{45} \mathrm{~A}$ large, unblinded, randomized, pragmatic trial in hospital-based outpatients in the United Kingdom identified 1721 patients for whom CBZ was considered standard treatment (ie, those with partial onset seizures) and randomized them to treatment with one of five AEDs: CBZ, gabapentin (GBP), lamotrigine (LTG), oxcarbazepine (OXC), or topiramate (TPM). Two primary outcome measures were used, ie, time to treatment failure and time to 12-month remission. LTG was significantly better than CBZ, GBP, and TPM and had a nonsignificant advantage over OXC in the time to treatment failure measure, but CBZ was significantly better than GBP and had a nonsignificant advantage over LTG, TPM, and OXC in time to 12-month remission. ${ }^{46}$ About $50 \%$ of patients reported side effects at

Table 3 AEDs available for partial onset seizures

\begin{tabular}{ll}
\hline Classical AEDs & New AED \\
\hline phenobarbital & felbamate \\
phenytoin & gabapentin \\
primidone & lamotrigine \\
carbamazepine & topiramate \\
valproate & tiagabine \\
& levetiracetam \\
& oxcarbazepine \\
& zonisamide \\
& pregabalin \\
& lacosamide \\
& vigabatrin \\
\hline
\end{tabular}

Notes: Drugs are listed in the order of approval in the US. Abbreviation: AEDs, antiepileptic drugs. 
some point in the study, but differences among AEDs were not large. Tiredness, headache, and fatigue were common symptoms for all drugs. Depression, disturbance of memory, and various psychiatric symptoms were also common, particularly with TPM. Rash was most common with CBZ and OXC, and least common with LTG. No multiple drug comparison trials have been done in patients with refractory complex partial seizures. A joint committee of the American Academy of Neurology and the American Epilepsy Society reviewed seven new AEDs (GBP, LTG, TPM, tiagabine [TGB], OXC, levetiracetam [LEV], and zonisamide [ZNS]) and considered all to be appropriate for adjunctive treatment of refractory partial seizures in adults. ${ }^{47}$ Multiple individual drug comparisons have failed to show consistent superiority of the new AEDs over the classical AEDs or of one new AED over another in the treatment of either new onset or refractory partial onset seizures. Wilby et al conducted a systematic review of 212 studies of newer drugs for epilepsy (GBP, LTG, LEV, OXC, TGB, TPM, and vigabatrin). They concluded that there is “ . .. little good-quality evidence from clinical trials to support the use of newer monotherapy or adjunctive therapy AEDs over older drugs, or to support the use of one newer AED in preference to another. In general, data relating to clinical effectiveness, safety and tolerability [have] failed to demonstrate consistent and statistically significant differences between the drugs". ${ }^{4}$

The current state of the evidence, therefore, is that none of the presently available AEDs is clearly superior to other AEDs in the management of refractory complex partial seizures. Therefore, the choice of which drug to use in an individual patient has to be based on other considerations, including previous success or failure of a given drug, cost, and side effect profile of the drug matched to individual patient characteristics (Table 4). For instance, drugs associated with significant weight gain potential, valproate (VPA) and pregabalin in particular, might best be avoided in already overweight individuals. Drugs with significant potential for cognitive impairment (eg, TPM) should be used with caution in individuals with intellectually demanding occupations. AEDs that can be taken once daily (eg, PHT, ZNS, TPM, and extended-release forms of CBZ, LTG, and LEV) have a clear advantage in patients for whom denial of their epilepsy can frequently lead to poor compliance. In women of child-bearing potential, AEDs with a significant risk of teratogenicity (eg, VPA) should be avoided.

There are a number of strategies for the optimal use of AEDs in the management of refractory complex partial seizures (Table 5). Diagnosis and classification of the
Table 4 Factors to consider when choosing an AED for a specific patient

\begin{tabular}{ll}
\hline Adverse reactions & AED(s) \\
\hline Hirsutism, gum hyperplasia & PHT \\
Alopecia, tremor & VPA \\
Weight gain & VPA, GBP, PGB \\
Weight loss & FBM, TPM, ZNS \\
Hyponatremia & CBZ, OXC \\
Teratogenicity & VPA \\
\hline Compliance issues & AED(s) \\
Once-daily dosing possible & PB, PHT,TPM, ZNS \\
& Extended-release AEDs \\
\hline
\end{tabular}

Abbreviations: AEDs, antiepileptic drugs; CBZ, carbamazepine; FBM, felbamate; GBP, gabapentin; OXC, oxcarbazepine; PB, phenobarbital; PBG, pregabalin; PHT, phenytoin;TPM, topiramate; VPA, valproate; ZNS, zonisamide.

seizure type and choice of AEDs have been discussed above. In the medically refractory patient, a review of previously tried AEDs is essential. Was the drug tried at maximal tolerated doses and given for long enough to achieve pharmacokinetic and pharmacodynamic steady states and to allow resolution of early treatment side effects, such as sedation and gastrointestinal upset? All too often potentially effective AEDs are abandoned because initial doses are so high or the rate of titration so fast that early side effects develop which could have been avoided had the drug been started more slowly. In the medically refractory patient, rapid titration of a new-to-the-patient AED is rarely necessary; the patient has had refractory seizures for a long time and a few days or even weeks spent slowly increasing the dose can often make the difference between success and failure.

Table 5 Strategies for optimizing management of refractory complex partial seizures

- Verify the diagnosis of epilepsy and determine the etiology if possible

- Review the patient's seizure descriptions to be sure of their correct classification

- Match choice of AED to the seizure type(s) and to the specific patient

- Use monotherapy if possible

- Use polytherapy if necessary

-When adding an AED start low and go slow, but, if necessary push to the maximum tolerated dose

- Consider changing the timing of dosing to reduce toxicity

- Use pharmacokinetic principles to fine-tune the dose

- Adjust doses for drug-drug interactions

- Do not give up

Abbreviation: AED, antiepileptic drug. 
If possible, seizure control with a single agent is preferable. This avoids the potential for drug-drug interactions, is generally less expensive, and may result in better seizure control. ${ }^{49}$ Therefore, ideally, each of the first-line AEDs available for management of partial onset seizures (ie, all current first-line drugs except ethosuximide) which are appropriate for the specific patient, should be tried before considering polytherapy. Although one drug may fail as monotherapy, another may succeed, even when the two drugs have similar profiles in animal models and share the same mechanism of action. ${ }^{50}$ For example, when PHT and CBZ (both stabilizers of the sodium channel) were compared in three crossover trials in the treatment of complex partial seizures, some patients responded much better to one drug than to the other, even though there were no differences in the overall efficacy of the two drugs. ${ }^{51-53}$ However, sequential trials of AEDs as monotherapy must not leave the patient without drug treatment. A useful approach (Figure 1) is to increase the dose of a single AED to that which is maximally tolerated. If seizure control is not achieved, a second AED should be added and the dose should be raised gradually to mid-therapeutic range. Transient dose-related side effects are far less dangerous to the patient than an increase
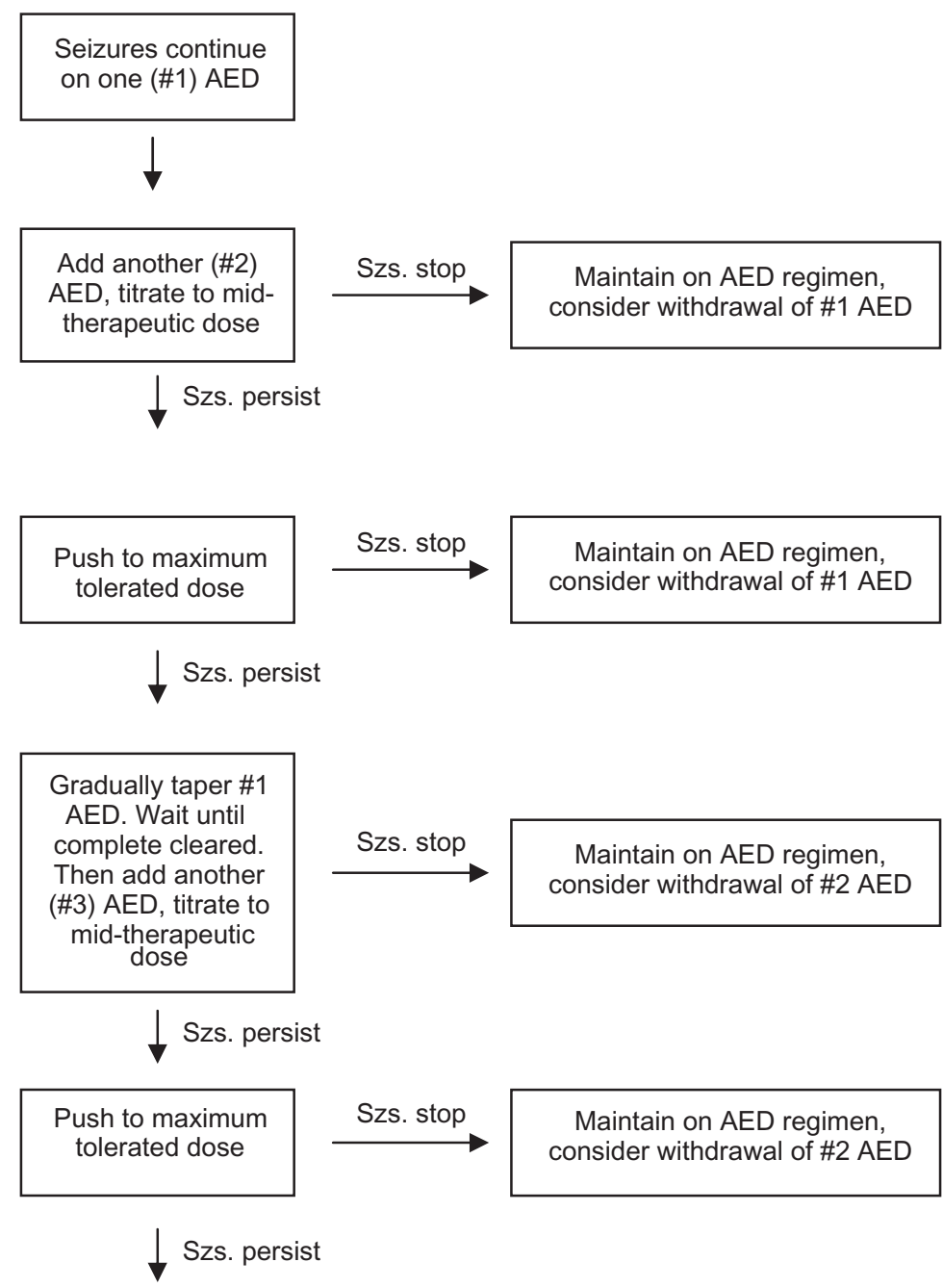

- Continue algorithm with additional AEDs, or

- Consider resective surgery, or, if not a candidate,

- Consider VNS or clinical trial of experimental AEDs

Figure I Algorithm for successive monotherapy trials in a patient with uncontrolled seizures. This approach allows testing the efficacy and tolerability of AEDs as monotherapy without subjecting the patient to the risk of increased seizures that could occur if an existing drug is tapered simultaneously with the uptitration of an additional drug. Dose-related side effects are in general much less dangerous than the risk of additional seizures and can be treated by lowering the dose of the offending drug. Abbreviations: AED, antiepileptic drug; Szs, seizures; VNS, vagal nerve stimulator. 
in seizure frequency or severity that could occur if the dose of the first drug is lowered before the dose of the second drug is increased. If there is at least some therapeutic response to the second drug, the dose can be escalated further until either seizure control is achieved or unacceptable side effects appear. If seizure control is achieved, the dose of the first drug then may be gradually tapered to determine if seizure control can be maintained with monotherapy. If there is no response, the procedure can be repeated with yet another AED. In this way a series of AEDs used as monotherapy and as dual therapy can be screened to identify the optimal drug or combination of drugs for a particular patient..$^{50}$ Although combination therapy has little additional benefit in most patients with new onset epilepsy, ${ }^{17}$ it may have significant benefit in patients with refractory complex partial seizures. At least one-third of patients with new onset epilepsy and more than half with complex partial seizures will require more than one drug to achieve seizure control.

The use of more than one AED in a patient creates the potential for pharmacokinetic drug-drug interactions, as does the use of AEDs in patients taking drugs for other indications. Most of the "classical" AEDs have the potential for either induction or inhibition of cytochrome P450 isozymes; fortunately many of the newer drugs do not. Table 6 provides a list of currently available AEDs and their potential for drug-drug interactions. Table 7 provides a list of drugs other than AEDs which may interact with AEDs. These potential interactions have to be considered whenever a patient is taking more than one drug, and may necessitate adjusting the dose of one AED if others are also being prescribed, or if the patient is taking other drugs as well as AEDs. Grapefruit inhibits CYP3A4, a P450 isozyme important in the metabolism of some of the benzodiazepines, CBZ, TGB, and ZNS..$^{54}$

Table 6 Potential drug-drug interactions of AEDs caused by microsomal P450 induction or inhibition

\begin{tabular}{lll}
\hline Inducers & Inhibitors & No effect \\
\hline carbamazepine & felbamate & gabapentin \\
phenobarbital & $\begin{array}{c}\text { valproate } \\
\text { zonisamide } \\
\text { phenytoin }\end{array}$ & $\begin{array}{l}\text { lacosamide } \\
\text { lamotrigine }\end{array}$ \\
$\begin{array}{l}\text { primidone } \\
\begin{array}{l}\text { oxcarbazepine (estrogens, } \\
\text { dihydropyridines, cyclosporin) }\end{array}\end{array}$ & \\
$\begin{array}{l}\text { vigabatrin } \\
\text { zonisamide (min. carbamazepine) }\end{array}$ & & $\begin{array}{l}\text { levetiracetam } \\
\text { pregabalin } \\
\text { tiagabine }\end{array}$ \\
\hline
\end{tabular}

Abbreviation: AEDs, antiepileptic drugs.
Table 7 Potential effects of some nonantiepileptic drugs on AED elimination via microsomal P450 enzyme

\begin{tabular}{lll}
\hline Inducers & Inhibitors & \\
\hline alcohol & allopurinol & erythromycin \\
nicotine & chloramphenicol & grapefruit \\
oral contraceptives & cimetidine & isoniazid, PAS \\
other steroids & coumarin & phenylbutazone \\
phenothiazines & diltiazem & propranolol \\
rifampin & (and other CCBs) & propoxyphene \\
& disulfiran & sulfonamides \\
\hline
\end{tabular}

Abbreviations: AED, antiepileptic drug; CCBs, calcium channel blockers; PAS, para-aminosalicylic acid.

It is essential in the management of the patient with refractory complex partial seizures to push the dose(s) of the AED or combination of drugs as high as necessary to achieve seizure control without producing unacceptable side effects. Animal and human studies show that seizure control is correlated with AED concentrations. ${ }^{50}$ The therapeutic range for all currently available AEDs is no more than an empiricallyderived probability statement, with the low end being the serum concentration at which efficacy is seen in most but not all patients and the high end being the concentration at which toxicity is seen in most but not all patients. These therapeutic ranges have been determined empirically in a nonsystematic fashion. There has never been a formal prospective determination of the median effective dose or median toxic dose of any AED in human patients. Therefore, the effective dose and the toxic dose of an AED must be determined empirically for individual patients. The therapeutic range should only serve as a rough guide to dosing, but should never inhibit upward titration of the dose of an AED if the patient is tolerating the drug well and continues to have seizures.

The pharmacokinetic characteristics of the drug must be considered when increasing the dose of an AED. Most AEDs exhibit first-order kinetics within the therapeutic range, so that there is a linear relationship between dose and serum concentration (Figure 2). However, three current AEDs (CBZ, VPA, GBP) exhibit a curvilinear relationship between dose and concentration such that there is a decrease in the concentration response as the dose is increased. On the other hand PHT exhibits Michaelis-Menten (saturation) elimination kinetics within the therapeutic range, and thus there is an increase in the concentration response as the dose is increased. Small increases in dose result in large increases in concentration. Because PHT is still used by many physicians (including non-neurologists) it is important to understand how to make adjustments of the dose to achieve maximum seizure control whilst avoiding toxic side effects. At the high 


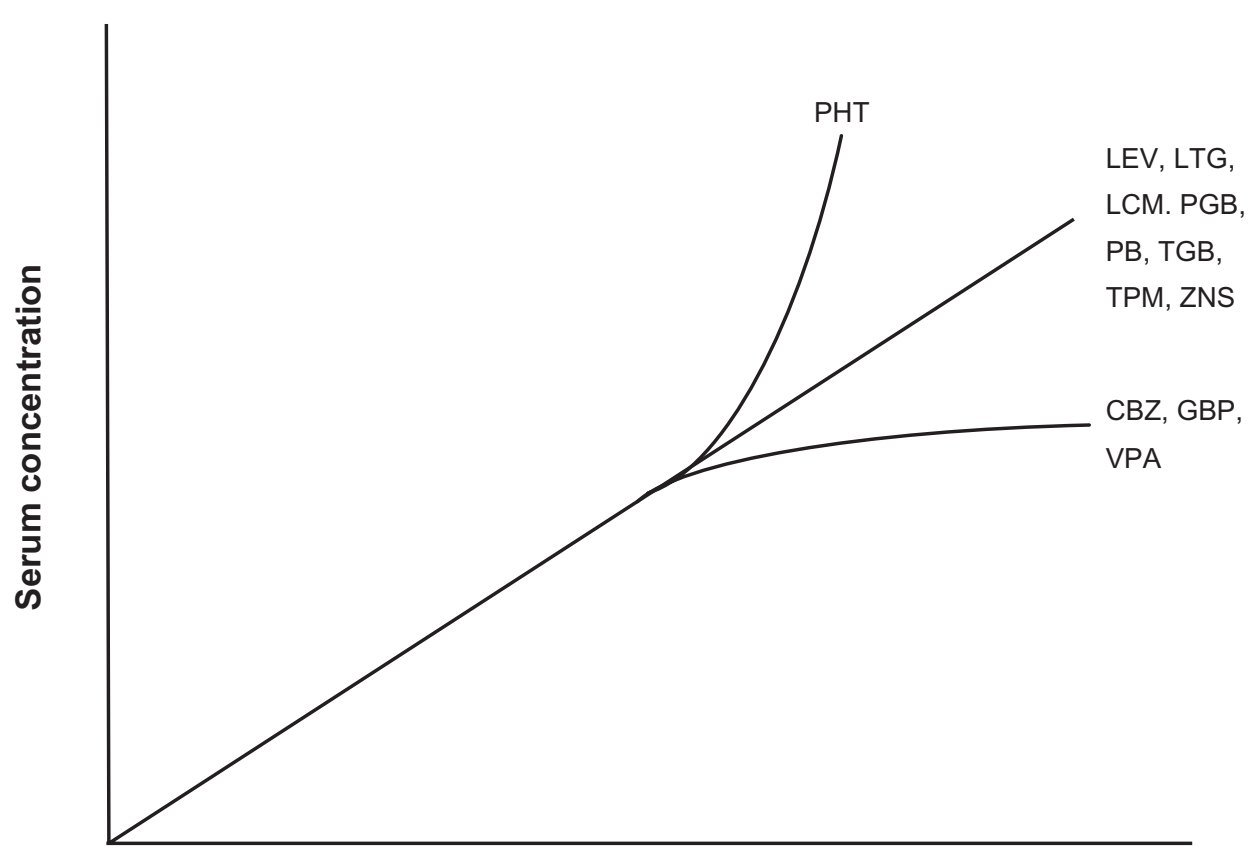

Maintenance dose

Figure 2 Effect of dose on elimination kinetics of AEDs.

For most AEDs the relationship between AED dose and concentration is linear. However, valproate (because of saturation of serum protein-binding sites), gabapentin (because of saturation of L-amino acid transport enzymes in the intestine) and carbamazepine (because of autoinduction of its own metabolizing $\mathrm{p} 450$ isozymes) exhibit a nonlinear relationship between dose and serum concentration. The higher the dose, the less increase in concentration per unit dose is achieved. Phenytoin exhibits Michaelis-Menten (saturation) kinetics. Because of the saturation of the microsomal p450 isozymes responsible for phenytoin metabolism, the higher the dose the greater the increase in serum concentration per unit dose. Figure modified and updated from Kriel and Cloyd. ${ }^{66}$

Abbreviations: AED, antiepileptic drug; CBZ, carbamazepine; GBP, gabapentin; LEV, levetiracetam; LTG, lamotrigine; LCM, lacosamide; PGB, pregabalin; PHT, phenytoin; $\mathrm{PB}$, phenobarbital;TGB, tiagabine;TPM, topiramate;VPA, valproate; ZNS, zonisamide.

end of the therapeutic range PHT doses should be adjusted by $10 \mathrm{mg}$ increments to avoid excessive increases in serum concentration and consequent toxic side effects. ${ }^{50}$ Table 8 provides predicted increases in PHT serum concentration following $10 \mathrm{mg}$ increases in the daily dose for low, mid, and high serum concentrations. $10 \mathrm{mg}$ increments in the oral dose can be achieved for any dose of $180 \mathrm{mg}$ and above by using a combination of the available $100 \mathrm{mg}$ and $30 \mathrm{mg}$ capsules.

Table 8 Predicted increase in serum phenytoin concentration following a $10 \mathrm{mg}$ increase in daily dose ${ }^{50}$

\begin{tabular}{|c|c|c|c|}
\hline Daily dose & $200 \mathrm{mg}$ & $300-400 \mathrm{mg}$ & $500 \mathrm{mg}$ \\
\hline Initial concentration & \multicolumn{3}{|c|}{ Effect of a $10 \mathrm{mg}$ change in dose } \\
\hline $10 \mu \mathrm{g} / \mathrm{mL}$ & $1.5 \mu \mathrm{g} / \mathrm{mL}$ & $1.0 \mu \mathrm{g} / \mathrm{mL}$ & $0.5 \mu \mathrm{g} / \mathrm{mL}$ \\
\hline $15 \mu \mathrm{g} / \mathrm{mL}$ & $3.0 \mu \mathrm{g} / \mathrm{mL}$ & $\mathrm{I} .5 \mu \mathrm{g} / \mathrm{mL}$ & $0.75 \mu \mathrm{g} / \mathrm{mL}$ \\
\hline $20 \mu g / m L$ & $6.0 \mu \mathrm{g} / \mathrm{mL}$ & $3.0 \mu \mathrm{g} / \mathrm{mL}$ & $\mathrm{I} .5 \mu \mathrm{g} / \mathrm{mL}$ \\
\hline
\end{tabular}

Notes: *By extrapolating between concentrations, this table can be used to adjust the maintenance phenytoin dose for most patients for whom a steady-state phenytoin concentration is available. Some individuals, however, are hypometabolizers of phenytoin and some are hypermetabolizers. For such patients it may be necessary to shift to the left or right column, respectively, even if the patient is taking $300-400 \mathrm{mg} / \mathrm{day}$. The table was derived from a nomogram for adjustment of phenytoin maintenance doses created by Rambeck et al. ${ }^{67}$
Therapeutic drug monitoring can be useful in the management of refractory complex partial seizures. It is particulary useful in helping to determine which drug is causing adverse effects if a patient is taking two or more AEDs. It may also be useful in some circumstances to verify compliance. Finally, serum concentrations are very useful for fine-tuning PHT dosing, especially in the intensive care setting where a patient may be receiving a multitude of drugs, but also in the outpatient setting, as described above. Peak concentrations are most useful in assessing toxicity; trough levels are most helpful in assessing compliance. Serum concentrations are readily and rapidly available for most of the classical AEDs (PB, PHT, CBZ, VPA) but not as easily obtainable for most of the newer AEDs. Furthermore, therapeutic ranges are not nearly as well established for the newer AEDs, so therapeutic monitoring is less useful in the management of these drugs.

Sometimes seizure control can be achieved by changing the timing of AED dosing. As a generalization, an AED should not be given less often than at least once a half-life to avoid excessive fluctuations in peak and trough concentrations. For rapidly absorbed drugs (those without extended-release formulations) the peak concentration $\left(\mathrm{T}_{\text {max }}\right)$ 
occurs about 1.0 to 1.5 hours after oral administration. If the patient experiences post-dose toxicity, this can be reduced by using smaller doses more frequently throughout the day. However, if the drug has a long-half life, it may be possible to give the entire dose immediately before bedtime, so that the peak concentration occurs when the patient is in slow-wave sleep. Drugs that cause insomnia (felbamate and sometimes LTG), however, are best taken early in the day. It is clear that compliance is improved by once or twice a day dosing, and is reduced if AED dosing is more frequent, ${ }^{55}$ and this must also be considered in any dosing strategy.

Surgical strategies for the management of refractory complex partial seizures will be discussed below. However, if surgery is not an option, it is important to continue to try to achieve seizure control with AEDs. Do not give up. There are now 16 AEDs available in the US for treatment of refractory complex partial seizures. This means there are 120 potential two-drug combinations and many more three drug combinations. Perseverance alone can sometimes lead to successful control of refractory complex partial seizures. Luciano and Shorvon $^{56}$ recently reported the result of 265 drug additions in 155 adult patients with chronic pharmacoresistant epilepsy. Twenty-eight percent eventually became seizure-free, but only $17 \%$ stopped having seizures after the addition of only one AED. The other $11 \%$ required trials of two, three, or more additional AEDs to achieve seizure freedom. Similar results have been reported by Callaghan et al. ${ }^{57}$

\section{Surgical strategies}

Surgical intervention has to be a consideration in the management of refractory complex partial seizures. Currently available interventions include resection of the seizure focus, multiple subpial transection when the seizure focus is in eloquent cortex, destruction of the seizure focus by gamma knife, and implantation of a VNS. Deep brain stimulation and stimulation of the seizure focus are under study, but these are not yet approved interventions.

Patients should be considered for resective surgery when the seizure focus appears to be well localized and has a solo focus. This is best predicted by the seizure semiology at onset, as discussed above. The ideal candidate for resective surgery is the patient with refractory complex partial seizures whose semiology suggests mesial temporal onset, who has failed an adequate trial of at least two or three AEDs, and who exhibits mesial temporal sclerosis on MRI. In such patients, success rates of $70 \%-90 \%$ for selective amygdalohippocampectomy or temporal lobectomy can be expected in the hands of an experienced surgeon. Téllez-Zentano et $\mathrm{al}^{58}$ recently reviewed the long-term outcome of surgical procedures for epilepsy. Overall the median proportion of long-term seizure-free patients was $66 \%$ for temporal lobe resections, $46 \%$ for occipital and parietal resections, and $27 \%$ for frontal lobe resections. Multiple subpial transections only resulted in long-term seizure freedom in $16 \%$. Successful destruction of the seizure focus with gamma radiation in patients with mesial temporal epilepsy has been reported in several recent series, with long-term success rates comparable with those for resective surgery. However, the mean time to seizure control has been 12 months, in contrast with immediate seizure freedom following open surgery. ${ }^{59-61}$

When medical treatment options have been exhausted and resective surgery is not possible (usually because of bilateral mesial temporal-independent seizure foci), implantation of a VNS should be considered in the patient with medically refractory complex partial seizures. Overall response rates of $35 \%$ to $45 \%$ have been reported, ${ }^{62-64}$ but few patients become completely seizure-free, and almost all remain on AEDs. Nonetheless, a VNS can be of great benefit to some patients with refractory complex partial seizures. High stimulation parameters are more effective than low stimulation parameters, but result in more adverse reactions. ${ }^{65}$

\section{Conclusion}

Epilepsy is the third most common neurologic disorder in incidence, but the second most common neurologic disorder in prevalence. Because it affects all ages and because of the social stigma attached to epilepsy in many cultures, it has a disproportionately high impact on the quality of life of the patients it affects. Refractory complex partial seizures affect at least 7.5 million up to perhaps as many as 10 million people worldwide. Many advances have been made in the management of complex partial seizures, but one-third of afflicted patients continue to have uncontrolled seizures. Fortunately, there are a number of strategies that can improve control of such seizures, as discussed above, and a number of new approaches to management that are likely to come to fruition over the next several decades and result in significant improvement in the management of complex partial seizures.

\section{Disclosures}

Dr Treiman has, at various times, received research support from, served as a consultant for, and/or served on a speakers' bureau for Abbott, Astra, Athena, Carter-Wallace, Cyberonics, Dainippon, Eisai, Élan, GlaxoSmithKline, Ikano, Janssen, 
Johnson and Johnson, Lundbeck, Merrell Dow, Novartis, Organon, Ovation, Ortho-McNeil, Parke Davis, Pfizer, Roche, Sanofi, Syntex, UCB, Valeant, and Wyeth. The author is not aware of any conflicts of interest in this work.

\section{References}

1. World Health Organization. Epilepsy: Aetiology, epidemiology and prognosis. Available at: www.who.int/mediacentre/factsheets/fs165/en/. 2001.

2. Banerjee PN, Filippi D, Allen HW. The descriptive epidemiology of epilepsy - a review. Epilepsy Res. 2009;85:31-45.

3. Hauser WA, Annegers JF, Kurland LT. Incidence of epilepsy and unprovoked seizures in Rochester, Minnesota: 1935-1984. Epilepsia. 1993;34:453-468.

4. Sidenvall R, Forsgren L, Heijbel J. Prevalence and characteristics of epilepsy in children in northern Sweden. Seizure. 1996;5:139-146.

5. Forsgren L, Bucht G, Eriksson S, Bergmark L. Incidence and clinical characterization of unprovoked seizures in adults: A prospective population-based study. Epilepsia. 1996;37:224-229.

6. Jallon P, Goumaz M, Haenggeli C, Morabia A. Incidence of first epileptic seizures in the canton of Geneva, Switzerland. Epilepsia. 1997:38:547-552.

7. Olafsson E, Hauser WA, Ludvigsson P, Gudmundsson G. Incidence of epilepsy in rural Iceland: a population-based study. Epilepsia. 1996;37:951-955

8. Olafsson E, Ludvigsson P, Gudmundsson G, Hesdorffer D, Kjartansson O, Hauser WA. Incidence of unprovoked seizures and epilepsy in Iceland and assessment of the epilepsy syndrome classification: A prospective study. Lancet Neurol. 2005;4:627-634.

9. Farrell MA, DeRosa MJ, Curran JG, et al. Neuropathologic findings in cortical resections (including hemispherectomies) performed for the treatment of intractable childhood epilepsy. Acta Neuropathol. 1992;83:246-259.

10. Kuzniecky R, Murro A, King D, et al. Magnetic resonance imaging in childhood intractable partial epilepsies: Pathologic correlations. Neurology. 1993;43:681-687.

11. Wyllie E, Comair YG, Kotagal P, Bulacio J, Bingaman W, Ruggieri P Seizure outcome after epilepsy surgery in children and adolescents. Ann Neurol. 1998;44:740-748.

12. Frater JL, Prayson RA, Morris III HH, Bingaman WE. Surgical pathologic findings of extratemporal-based intractable epilepsy: A study of 133 consecutive resections. Arch Pathol Lab Med. 2000;124 545-549.

13. Pasquier B, Peoc'h M, Fabre-Bocquentin B, et al. Surgical pathology of drug-resistant partial epilepsy. A 10-year-experience with a series of 327 consecutive resections. Epileptic Disord. 2002;4:99-119.

14. Alving J. Classification of the epilepsies. An investigation of 1,508 consecutive adult patients. Acta Neurol Scan. 1978;58:205-212.

15. Banerjee PN, Hauser WA. Incidence and prevalence. In: Engel J Jr, Pedley TA, editors. Epilepsy: A Comprehensive Textbook. 2nd ed. Philadelphia,PA: Wolters Kluwer Lippincott Williams Wilkins; 2008. p. $45-56$.

16. Berg AT, Shinnar S. The risk of seizure recurrence following a first unprovoked seizure: A quantitative review. Neurology. 1991;41: 965-972.

17. Kwan P, Brodie MJ. Early identification of refractory epilepsy. $N$ Engl J Med. 2000;342:314-319.

18. Tallis R, Hall G, Craig I, Dean A. How common are epileptic seizures in old age? Age Ageing. 1991;20:442-448.

19. Hauser WA. Seizure disorders: The changes with age. Epilepsia. 1992;33(Suppl 4):S6-S14

20. Hopkins A, Shorvon S. Definitions and epidemiology of epilepsy. In Hopkins A, Shorvon S, Cascino G, editors. Epilepsy. 2nd ed. London: Chapman \& Hall Medical; 2005. p. 1-24.
21. Carpio A, Hauser WA. Epilepsy in the developing world. Curr Neurol Neurosci Rep. 2009;9:319-326.

22. Sander JW, Hart YM, Johnson AL, Shorvon SD. National General Practice Study of Epilepsy: Newly diagnosed epileptic seizures in a general population. Lancet. 1990;336:1267-1271.

23. Loiseau J, Loiseau P, Guyot M, Duche B, Dartigues JF, Aublet B. Survey of seizure disorders in the French southwest. I. Incidence of epileptic syndromes. Epilepsia. 1990;31:391-396.

24. Loiseau J, Loiseau P, Duche B, Guyot M, Dartigues JF, Aublet B. A survey of epileptic disorders in southwest France: seizures in elderly patients. Ann Neurol. 1990;27:232-237.

25. Salazar AM, Jabbari B, Vance SC, Grafman J, Amin D, Dillon JD. Epilepsy after penetrating head injury. I. Clinical correlates: A report of the Vietnam Head Injury Study. Neurology. 1985;35:1406-1414.

26. Jallon P, Loiseau P, Loiseau J. Newly diagnosed unprovoked epileptic seizures: Presentation at diagnosis in CAROLE study. Coordination Active du Reseau Observatoire Longitudinal de l'Epilepsie. Epilepsia. 2001;42:464-475.

27. Williamson PD, Engel J Jr. Anatomic classification of focal epilepsies. In: Engel J Jr, Pedley TA, editors. Epilepsy: A Comprehensive Textbook. 2nd ed. Philadelphia, PA: Wolters Kluwer Lippincott Williams \& Wilkins; 2008:2465-2477.

28. Hauser WA, Hesdorffer DC. Epilepsy Frequency, Causes and Consequences. 1st ed. New York, NY: Demos; 1990.

29. Loddenkemper T, Kotagal P. Lateralizing signs during seizures in focal epilepsy. Epilepsy Behav. 2005;7:1-17.

30. Maillard L, Vignal JP, Gavaret M, et al. Semiologic and electrophysiologic correlations in temporal lobe seizure subtypes. Epilepsia. 2004;45:1590-1599.

31. Commission on Classification and Terminology of the International League Against Epilepsy. Proposal for revised classification of epilepsies and epileptic syndromes. Epilepsia. 1989;30:389-399.

32. Rafal RD. Oculomotor functions of the parietal lobe: Effects of chronic lesions in humans. Cortex. 2006;42:730-739.

33. Deacon C, Wiebe S, Blume WT, McLachlan RS, Young GB, Matijevic S. Seizure identification by clinical description in temporal lobe epilepsy: How accurate are we? Neurology. 2003;61:1686-1689.

34. Chung SS, Gerber P, Kirlin KA. Ictal eye closure is a reliable indicator for psychogenic nonepileptic seizures. Neurology. 2006;66: $1730-1731$.

35. Salinsky M, Kanter R, Dasheiff RM. Effectiveness of multiple EEGs in supporting the diagnosis of epilepsy: An operational curve. Epilepsia. 1987;28:331-334

36. Devinsky O, Kelley K, Porter RJ, Theodore WH. Clinical and electroencephalographic features of simple partial seizures. Neurology. 1988;38:1347-1352

37. Bare MA, Burnstine TH, Fisher RS, Lesser RP. Electroencephalographic changes during simple partial seizures. Epilepsia. 1994;35: 715-720.

38. Sirven JI, Sperling MR, French JA, O'Connor MJ. Significance of simple partial seizures in temporal lobe epilepsy. Epilepsia. 1996;37:450-454

39. Cooper R, Winter AL, Crow HJ, Walter WG. Comparison of subcortical, cortical and scalp activity using chronic indwelling electrodes in man. Electroencephalogr Clin Neurophysiol. 1965;18:217-228.

40. Tao JX, Ray A, Hawes-Ebersole S, Ebersole JS. Intracranial EEG substrates of scalp EEG interictal spikes. Epilepsia. 2005;46:669-676.

41. Tao JX, Baldwin M, Hawes-Ebersole S, Ebersole JS. Cortical substrates of scalp EEG epileptiform discharges. J Clin Neurophysiol. 2007;24:96-100.

42. O'Brien TJ, So EL, Mullan BP, et al. Subtraction SPECT co-registered to MRI improves postictal SPECT localization of seizure foci. Neurology. 1999;52:137-146

43. O'Brien TJ, So EL, Mullan BP, et al. Subtraction peri-ictal SPECT is predictive of extratemporal epilepsy surgery outcome. Neurology. 2000;55:1668-1677. 
44. Mattson RH, Cramer JA, Collins JF, et al. Comparison of carbamazepine, phenobarbital, phenytoin, and primidone in partial and secondarily generalized tonic-clonic seizures. N Engl J Med. 1985;313:145-151.

45. Mattson RH, Cramer JA, Collins JF. A comparison of valproate with carbamazepine for the treatment of complex partial seizures and secondarily generalized tonic-clonic seizures in adults. The Department of Veterans Affairs Epilepsy Cooperative Study No. 264 Group. N Engl J Med. 1992;10;327(11):765-771.

46. Marson AG, Al-Kharusi AM, Alwaidh M, et al. The SANAD study of effectiveness of carbamazepine, gabapentin, lamotrigine, oxcarbazepine, or topiramate for treatment of partial epilepsy: An unblinded randomised controlled trial. Lancet. 2007;369:1000-1015.

47. French JA, Kanner AM, Bautista J, et al. Efficacy and tolerability of the new antiepileptic drugs II: Treatment of refractory epilepsy: Report of the Therapeutics and Technology Assessment Subcommittee and Quality Standards Subcommittee of the American Academy of Neurology and the American Epilepsy Society. Neurology. 2004;62:1261-1273.

48. Wilby J, Kainth A, Hawkins N, et al. Clinical effectiveness, tolerability and cost-effectiveness of newer drugs for epilepsy in adults: A systematic review and economic evaluation. Health Technol Assess. 2005;9:1-157, iii-iv.

49. Schmidt D. Reduction of two-drug therapy in intractable epilepsy. Epilepsia. 1983;24:368-376.

50. Treiman DM. Treatment strategies. In: Willmore LJ, editor. Generalized tonic-clonic seizures: Identification, classification, and treatment. Deerfield, IL: Discovery International; 1995:54-69.

51. Simonsen N, Zander-Olsen P, Kühl V, Lund M, Wendelboe J. A comparative controlled study between carbamazepine and diphenylhydantoin in psychomotor epilepsy. Epilepsia. 1976;17: 169-176.

52. Troupin A, Ojemann LM, Halpern L, et al. Carbamazepine - a doubleblind comparison with phenytoin. Neurology. 1977;27:511-519.

53. Kosteljanetz M, Christiansen J, Mouritzen A, et al. Carbamazepine vs phenytoin. A controlled clinical trial in focal motor and generalized epilepsy. Arch Neurol. 1979;36:22-24.

54. Levy RH, Mather GG. Metabolic enzymes and antiepileptic drug interactions. Adv Neurol. 1998;76:49-55.

55. Cramer J, Vachon L, Desforges C, Sussman NM. Dose frequency and dose interval compliance with multiple antiepileptic medications during a controlled clinical trial. Epilepsia. 1995;36:1111-1117.
56. Luciano AL, Shorvon SD. Results of treatment changes in patients with apparently drug-resistant chronic epilepsy. Ann Neurol. 2007;62: 375-381.

57. Callaghan BC, Anand K, Hesdorffer D, Hauser WA, French JA. Likelihood of seizure remission in an adult population with refractory epilepsy. Ann Neurol. 2007;62:382-389.

58. Tellez-Zenteno JF, Dhar R, Wiebe S. Long-term seizure outcomes following epilepsy surgery: A systematic review and meta-analysis. Brain. 2005;128:1188-1198

59. Regis J, Rey M, Bartolomei F, et al. Gamma knife surgery in mesial temporal lobe epilepsy: A prospective multicenter study. Epilepsia. 2004;45:504-515

60. Bartolomei F, Hayashi M, Tamura M, et al. Long-term efficacy of gamma knife radiosurgery in mesial temporal lobe epilepsy. Neurology. 2008;70:1658-1663.

61. Barbaro NM, Quigg M, Broshek DK, et al. A multicenter, prospective pilot study of gamma knife radiosurgery for mesial temporal lobe epilepsy: Seizure response, adverse events, and verbal memory. Ann Neurol. 2009;65:167-175.

62. Ben-Menachem E, Manon-Espaillat R, Ristanovic R, et al. Vagus nerve stimulation for treatment of partial seizures: 1 . A controlled study of effect on seizures. Epilepsia. 1994;35:616-626.

63. Handforth A, DeGiorgio CM, Schachter SC, et al. Vagus nerve stimulation therapy for partial-onset seizures: A randomized activecontrol trial. Neurology. 1998;51:48-55.

64. DeGiorgio CM, Schachter SC, Handforth A, et al. Prospective longterm study of vagus nerve stimulation for the treatment of refractory seizures. Epilepsia. 2000;41:1195-1200.

65. Privitera MD, Welty TE, Ficker DM, Welge J. Vagus nerve stimulation for partial seizures. Cochrane Database Syst Rev. 2002; CD002896.

66. Kriel RL, Cloyd JC. Antiepilepsy drug therapy in children: Pharmacokinetcs, adverse effects, and monitoring. In: Swaiman KF, editor. Pediatric Neurology: Principles and Practice. St. Louis, MO: Mosby; 1994:583-609.

67. Rambeck B, Boenigk HE, Dunlop A, Mullen PW, Wadsworth J, Richens A. Predicting phenytoin dose - A revised nomogram. Ther Drug Monit. 1979;1:325-333.
Neuropsychiatric Disease and Treatment

\section{Publish your work in this journal}

Neuropsychiatric Disease and Treatment is an international, peerreviewed journal of clinical therapeutics and pharmacology focusing on concise rapid reporting of clinical or pre-clinical studies on a range of neuropsychiatric and neurological disorders. This journal is indexed on PubMed Central, the 'PsycINFO' database and CAS, and is the official

\section{Dovepress}

journal of The International Neuropsychiatric Association (INA). The manuscript management system is completely online and includes a very quick and fair peer-review system, which is all easy to use. Visit http://www.dovepress.com/testimonials.php to read real quotes from published authors. 